\title{
Tendência de mudança nas indicações de transplante penetrante de córnea
}

\author{
Trends in the indications for penetrating keratoplasty
}

\author{
Fabiana Tiemi Sano ${ }^{1}$ \\ Paulo Elias Correa Dantas ${ }^{2}$ \\ Wilmar Roberto Silvino ${ }^{3}$ \\ Janaina Zamuner Sanchez ${ }^{4}$ \\ Ronaldo Yuiti Sano ${ }^{5}$ \\ Fabio Adams ${ }^{6}$ \\ Maria Cristina Nishiwaki-Dantas ${ }^{7}$
}

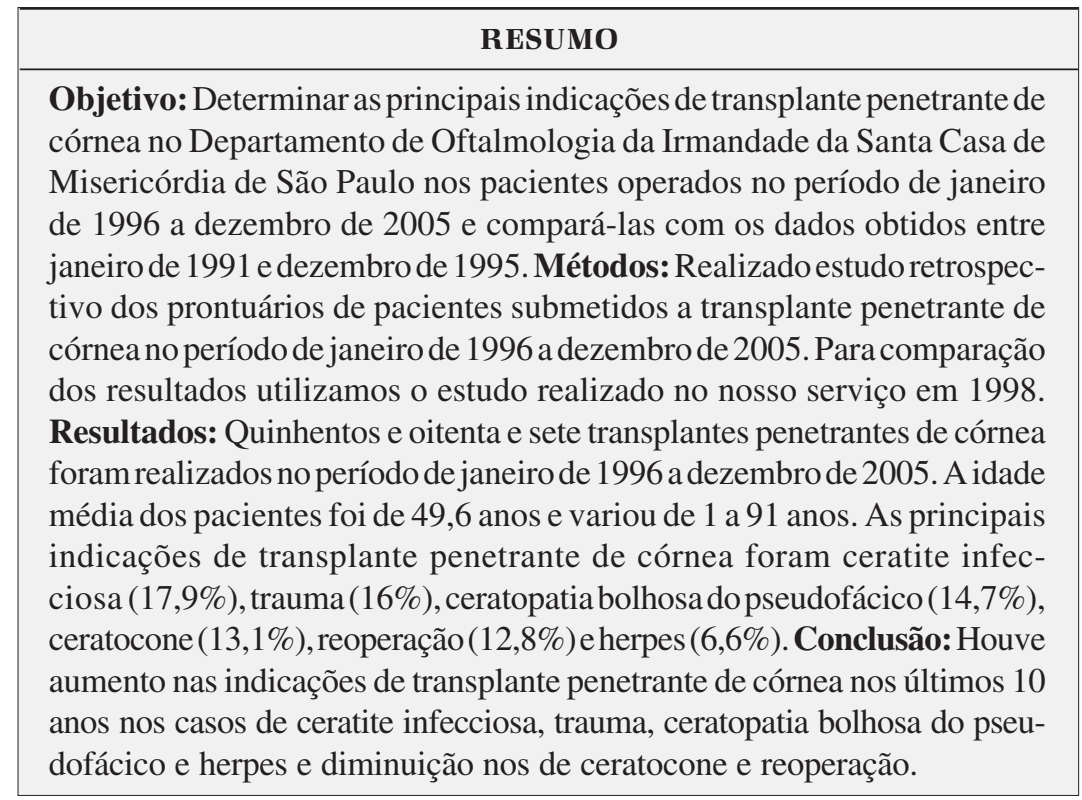

Descritores: Transplante de córnea/estatística \& dados numéricos; Córnea; Ceratoplastia penetrante; Tendência

\section{INTRODUCÃO}

O transplante de córnea consiste na substituição de córnea opaca ou doente por córnea doadora sadia. Essa substituição de tecido pode ser de espessura total (penetrante) ou parcial (lamelar ou lamelar profunda) ${ }^{(1)}$. Dependendo de sua finalidade, o transplante penetrante de córnea pode ser classificado em óptico, tectônico, terapêutico e cosmético. O transplante óptico é usado com o objetivo de melhorar a acuidade visual pela substituição do tecido corneano opaco por tecido doador transparente; o tectônico, de restaurar ou preservar a anatomia corneana em olhos com graves alterações estruturais; o terapêutico, de remover o tecido corneano inflamado ou infectado, que não responda à terapia clínica convencional, e o cosmético, a fins de melhorar a aparência do olho ${ }^{(2)}$.

As estatísticas do Eye Bank Association of América (EBAA) indicam que, nos Estados Unidos da América (EUA), são realizados mais de 35.000 transplantes de córnea anualmente ${ }^{(2)}$. No Brasil, segundo a Associação Brasileira de Transplante de Órgãos (ABTO), em 2005 foram realizados 8.713 transplantes de córnea, 2.181 transplantes a mais que em $2004^{(3)}$.

O progressivo aumento no número de transplantes de córnea realizados pode dever-se a alguns fatores, tais como melhor organização dos Bancos 
de Olhos (que proporciona maior captação e preservação de córneas), melhor seleção do tecido doado e os avanços da farmacologia, imunologia e microcirurgias oculares, que permitem realização de transplantes em situações antes consideradas inoperáveis ${ }^{(4)}$.

As principais indicações podem variar de acordo com o período de tempo estudado e do local de obtenção dos dados. Nos EUA, o ceratocone e os retransplantes eram considerados as principais indicações ${ }^{(5-6)}$. Com o advento das extrações de catarata pela técnica de facoemulsificação com implante de lente intra-ocular, a ceratopatia bolhosa do pseudofácico (CBPF) passou a ser a mais freqüente ${ }^{(7-12)}$. Nos diversos estudos brasileiros, o ceratocone ainda desponta como primeira indicação de transplante de córnea ${ }^{(4,13-20)}$.

Baseado em pressupostos de que hoje há maior número de transplantes de córnea efetuados no país e de que nosso volume cirúrgico crescente, objetivamos com este estudo determinar se houve mudança nas principais indicações de transplante penetrante de córnea no Departamento de Oftalmologia da Irmandade da Santa Casa de Misericórdia de São Paulo (ISCMSP), entre os pacientes operados no período de janeiro de 1996 a dezembro de 2005 , e os dados coletados entre janeiro de 1991 e dezembro de $1995^{(19)}$.

\section{MÉTODOS}

Foram estudados, retrospectivamente, prontuários de pacientes submetidos a transplante penetrante de córnea no Departamento de Oftalmologia da ISCMSP, no período de janeiro de 1996 a dezembro de 2005.

Os dados analisados foram sexo, raça, idade na ocasião da cirurgia e diagnóstico pré-operatório.

Para facilitar a comparação dos resultados com estudos prévios, as diversas categorias diagnósticas foram organizadas de acordo com a classificação proposta por Lindquist et al. em $1991^{(21)}$, de modo que as seguintes condições foram agrupadas sob a denominação de retransplante (falência endotelial primária, rejeição imunológica, glaucoma, ceratite infecciosa perfurada, recidiva de herpes, recidiva de úlcera reumática, $\mathrm{CBPF}$, rotura traumática e defeito epitelial crônico estéril).

Nesses casos, foram analisadas as causas imediatas e a doença primária responsável pelo primeiro transplante.

Com o auxílio da estatística descritiva, os resultados foram comparados com os de Nishiwaki-Dantas et al. ${ }^{(19)}$.

\section{RESULTADOS}

\section{Amostra estudada (prontuários)}

Quinhentos e oitenta e sete transplantes penetrantes de córnea foram realizados no período de janeiro de 1996 a dezembro de 2005. A média de idade foi de 49,6 $\pm 21,1$ anos (variou de 1 a 91 anos). Trezentos e doze (53,2\%) pacientes eram do sexo feminino e $275(46,8 \%)$ do masculino. Houve predomínio da raça branca, com $436(74,3 \%)$ pacientes, em relação à negra com $148(25,2 \%)$ e amarela com $3(0,5 \%)$ pacientes.

A indicação mais freqüente de transplante de córnea foi a ceratite infecciosa (bacteriana e fúngica), com 17,9\% dos casos (Tabela 1), o que engloba os transplantes terapêuticos realizados por ceratite infecciosa ativa ( $8,5 \%$ dos casos), os transplantes ópticos por leucoma corneano após infecção $(5,3 \%)$ e cicatriz corneana secundária a tracoma $(4,1 \%)$ (Tabela 1$)$.

A segunda causa mais comum de transplante de córnea foi trauma, com 94 casos (16\%) (Tabela 1).

A CBPF foi a terceira indicação mais freqüente $(14,7 \%)$, seguida pelo ceratocone $(13,1 \%)$ (Tabela 1$)$.

Os retransplantes representaram 12,8\% dos casos (Tabela 1). A indicação do primeiro transplante (antes do retransplante) mais freqüente foi $\operatorname{CBPF}(29,3 \%)$, seguida por trauma $(25,3 \%)$, ceratite infecciosa $(14,7 \%)$, herpes $(9,3 \%)$, ceratocone $(9,3 \%)$, distrofia de Fuchs (4\%), queimadura (4\%), ceratite ulcerativa não infecciosa $(2,7 \%)$ e descolamento da membrana de Descemet $(1,3 \%)$ (Tabela 2$)$.

Falência endotelial primária $(48,0 \%)$ e rejeição imunológica $(37,3 \%)$ foram as principais causas imediatas do retransplante (Tabela 3).

As demais causas de transplante penetrante de córnea são citadas na tabela 1 .

\begin{tabular}{|c|c|c|c|c|}
\hline \multirow[b]{2}{*}{ Indicações } & \multicolumn{2}{|c|}{ 1991-1995 } & \multicolumn{2}{|c|}{ 1996-2005 } \\
\hline & № & $\%$ & № & $\%$ \\
\hline Ceratocone & 54 & 21,7 & 77 & 13,1 \\
\hline Reoperação & 44 & 17,7 & 75 & 12,8 \\
\hline Ceratite infecciosa & 37 & 14,9 & 105 & 17,9 \\
\hline Ativa & 11 & 4,5 & 50 & 8,5 \\
\hline Inativa & 16 & 6,4 & 31 & 5,3 \\
\hline Tracoma & 10 & 4,3 & 24 & 4,1 \\
\hline Trauma & 21 & 8,4 & 94 & 16,0 \\
\hline Ceratopatia bolhosa do pseudofácico & 20 & 8,0 & 86 & 14,7 \\
\hline Distrofia não Fuchs & 16 & 6,4 & 18 & 3,1 \\
\hline Ceratite ulcerativa não infecciosa & 10 & 4,0 & 12 & 2,0 \\
\hline Distrofia de Fuchs & 9 & 3,6 & 17 & 2,9 \\
\hline Herpes & 9 & 3,6 & 39 & 6,6 \\
\hline Vírus não herpes & 6 & 2,4 & 0 & 0 \\
\hline Ceratopatia bolhosa afácica & 5 & 2,0 & 9 & 1,5 \\
\hline Queimadura & 3 & 1,2 & 21 & 3,6 \\
\hline Anomalia congênita & 2 & 0,8 & 0 & 0 \\
\hline Degeneração & 1 & 0,4 & 1 & 0,2 \\
\hline Desconhecida & 1 & 0,4 & 0 & 0 \\
\hline Glaucoma & 7 & 2,8 & 8 & 1,4 \\
\hline Uveíte & 2 & 0,8 & 0 & 0 \\
\hline $\begin{array}{l}\text { Descolamento da membrana } \\
\text { de Descemet }\end{array}$ & 1 & 0,4 & 19 & 3,2 \\
\hline Cirurgia refrativa & 1 & 0,4 & 0 & 0 \\
\hline Outros & 0 & 0 & 6 & 1,0 \\
\hline Total & 249 & 99,9 & 587 & 100 \\
\hline №= número; $\%=$ porcentagem & & & & \\
\hline
\end{tabular}




\begin{tabular}{|c|c|c|c|c|}
\hline \multirow[b]{2}{*}{ Indicações } & \multicolumn{2}{|c|}{ 1991-1995 } & \multicolumn{2}{|c|}{ 1996-2005 } \\
\hline & № & $\%$ & № & $\%$ \\
\hline Trauma & 9 & 20,4 & 19 & 25,3 \\
\hline Ceratite infecciosa & 8 & 18,2 & 11 & 14,7 \\
\hline Herpes & 6 & 13,6 & 7 & 9,3 \\
\hline Ceratite ulcerativa não infecciosa & 6 & 13,6 & 2 & 2,7 \\
\hline Ceratopatia bolhosa do pseudofácico & 5 & 11,4 & 22 & 29,3 \\
\hline Ceratocone & 3 & 6,8 & 7 & 9,3 \\
\hline Ceratopatia bolhosa afácica & 2 & 4,5 & 0 & 0 \\
\hline Distrofia de Fuchs & 2 & 4,5 & 3 & 4,0 \\
\hline Queimadura & 1 & 2,3 & 3 & 4,0 \\
\hline Anomalia congênita & 1 & 2,3 & 0 & 0 \\
\hline $\begin{array}{l}\text { Descolamento da membrana } \\
\text { de Descemet }\end{array}$ & 0 & 0 & 1 & 1,3 \\
\hline Total & 43 & 99,8 & 75 & 99,9 \\
\hline
\end{tabular}

\begin{tabular}{|c|c|c|c|c|}
\hline \multirow[b]{2}{*}{ Causas } & \multicolumn{2}{|c|}{ 1991-1995 } & \multicolumn{2}{|c|}{$1996-2005$} \\
\hline & № & $\%$ & № & $\%$ \\
\hline Falência endotelial primária & 17 & 38,6 & 36 & 48,0 \\
\hline Rejeição imunológica & 15 & 34,1 & 28 & 37,3 \\
\hline Glaucoma & 4 & 9,1 & 1 & 1,3 \\
\hline Ceratite infectada perfurada & 2 & 4,5 & 6 & 8,0 \\
\hline Recidiva de herpes & 2 & 4,5 & 1 & 1,3 \\
\hline Recidiva de úlcera reumática & 1 & 2,3 & 0 & 0 \\
\hline Ceratopatia bolhosa do pseudofácico & 1 & 2,3 & 3 & 4,0 \\
\hline Rotura traumática & 1 & 2,3 & 0 & 0 \\
\hline Defeito epitelial crônico estéril & 1 & 2,3 & 0 & 0 \\
\hline Total & 44 & 100 & 75 & 99,9 \\
\hline
\end{tabular}

\section{Comparação com Nishiwaki-Dantas e colaboradores}

A média de idade que era de 42,6 $\pm 3,9$ (variou de 1 a 89 anos) $)^{(19)}$ passou para 49,6 $\pm 21,1$ (variou de 1 a 90 anos) $(\mathrm{p}<0,0001)$.

Houve aumento na freqüência de transplante de córnea realizados nos casos de ceratite infecciosa (bacteriana e fúngica) (de 14,9\% para 17,9\%), principalmente nos casos de infecção ativa (de $4,5 \%$ para $8,5 \%$ ) (Tabela 1 ).

Houve também aumento nos casos de trauma (de 8,4\% para $16,0 \%$ ) e CBPF (de $8,0 \%$ para $14,7 \%$ ) e diminuição nos casos de ceratocone (de 21,7\% para $13,1 \%$ ) (Tabela 1).

Retransplante, que representava $17,7 \%$ do total de transplantes, diminuiu sua freqüência para 12,8\% (Tabela 1).

Nos casos de retransplante, CBPF passou a ser a principal indicação do primeiro transplante (de $13,6 \%$ para $29,3 \%$ ) (Tabela 2).
Falência endotelial primária e rejeição imunológica foram as principais causas de retransplante em ambos os estudos (Tabela 3).

\section{DISCUSSÃO}

Na amostra estudada, houve predomínio dos transplantes penetrantes de córnea realizados nos pacientes do sexo feminino $(53,2 \%)$ e da raça branca $(74,3 \%)$, assim como no estudo de 1998 (52,2\% dos pacientes do sexo feminino e $76,1 \%$ da raça branca $)^{(19)}$.

Ceratite infecciosa foi a principal indicação de transplante penetrante de córnea $(17,9 \%)$, o que diverge da maioria dos estudos que apontam a CBPF e o ceratocone como as indicações mais freqüentes ${ }^{(4,7-9,11-15,17-18,20,22-25)}$. Nos estudos realizados nos países em desenvolvimento, como China e Índia, a freqüência de transplante de córnea por ceratite infecciosa também é alta ${ }^{(26-28)}$. Em relação ao estudo anterior (pacientes operados entre 1991 e 1995) ${ }^{(19)}$, observou-se aumento na frequiência de transplantes de córnea por ceratite infecciosa (para comparação desses dados vide tabela 2), principalmente dos realizados nos casos de ceratite infecciosa ativa (de 4,4\% no estudo anterior para $8,5 \%$ no estudo atual).

A partir de maio de 2000, com a criação das Organizações de Procura de Órgãos (OPO) e o Sistema de Lista Única para Transplantes de Córnea - Organização e Procura de Córneas (OPC), pelo Ministério da Saúde, os bancos de olhos passaram a ser normatizados. Antes da criação das OPCs, as córneas captadas eram preservadas e utilizadas exclusivamente na instituição ${ }^{(29-30)}$. Após maio de 2000, as córneas captadas e preservadas na instituição passaram a ser encaminhadas para uma central, que dentre outras funções redistribui as córneas para os diversos serviços que fazem parte da lista única (que incluem instituições que não são captadoras de córnea). Considerando que no início da implementação de tal sistema não houve aumento no número de doações e captações, o Banco de Olhos da Santa Casa passou a dispor de menor número de córneas para uso próprio.

Desta forma, segundo dados obtidos nos arquivos do Banco de Olhos da ISCMSP, nos primeiros dois anos após a criação das OPCs, houve diminuição dos transplantes penetrantes de córnea realizados com finalidade óptica. Em 1999, foram realizados $81(81,8 \%)$ transplantes ópticos e $18(18,2 \%)$ terapêutico-tectônicos. Em 2000, foram 10 (27,0\%) transplantes ópticos e em 2001 apenas 9 (15,8\%), enquanto os transplantes tectônicos-terapêuticos aumentaram para $27(73,0 \%)$ em 2000 e 48 (84,2\%) em 2001.

Nossa instituição é um hospital de referência terciária e recebe muitos casos graves. Esses, por serem considerados prioridade, não aguardam na fila de transplante, o que também contribui para o aumento dos transplantes terapêutico-tectônicos de córnea.

Trauma foi a segunda causa mais freqüente de indicação de transplante de córnea (16\%), com aumento de 7,6\% em 
relação ao estudo anterior ${ }^{(19)}$. Nos diversos estudos analisados, os valores variaram de $0,9 \%$ a $7,91 \%{ }^{(12,22-23,27,31-32)}$.

Apesar da principal indicação de transplante de córnea nos EUA ser a $\mathrm{CBPF}^{(7-9,11-12,25)}$, estudos mostram que nos últimos anos há tendência a diminuir o número de $\operatorname{casos}^{(8,23,33)}$. Em nosso estudo, a freqüência aumentou de $8 \%$ para $14,7 \%$ e representa atualmente a terceira indicação. O aumento do número de casos de descompensação corneana após cirurgia de catarata pode ser atribuído ao aumento do número de cirurgias de catarata realizadas no serviço (foram realizadas 2.300 cirurgias de catarata no período de janeiro de 1991 a dezembro de 1995 e 15.753 cirurgias no período de janeiro de 1996 a dezembro de 2005), com conseqüente aumento das complicações associadas. Como a catarata é mais freqüente em pacientes com idade superior a 60 anos, isso poderia explicar o aumento da idade média no estudo atual. Estes dados foram obtidos do Serviço de Arquivo Médico e Estatística (SAME) da Irmandade da Santa Casa de São Paulo. Houve também aumento do número de transplantes realizados por CBPF no Hospital das Clínicas de São Paulo ${ }^{(14)}$, no Hospital de Florianópolis ${ }^{(17)}$ e na Faculdade de Medicina da Fundação $\mathrm{ABC}^{(13)}$.

Ceratocone continua sendo uma das principais indicações de transplante de córnea na América Latina e na Euro$\mathrm{pa}^{(4,13-20,22-23,25)}$. Neste estudo, foi a quarta indicação mais freqüente, com tendência a queda nos últimos dez anos. Fato similar foi relatado por outros autores ${ }^{(33-34)}$, que atribuíram o menor número de portadores desta doença submetidos a transplante de córnea à melhor adaptação e qualidade das lentes de contato em pacientes com ceratocone.

Houve aumento dos retransplantes de córnea (de 12,8\% para $17,7 \%)$. As indicações mais freqüentes do primeiro transplante foram as condições corneanas associadas a inflamação, como CBPF, ceratite infecciosa (bacteriana e fúngica), herpes e as responsáveis por maior alteração estrutural, como nos casos de trauma (Tabela 2). O aumento de retransplantes por CBPF (de $11,4 \%$ para $29,3 \%$ ) também pode estar relacionado ao aumento de cirurgias de catarata realizadas nos últimos anos. Assim como em estudos anteriores ${ }^{(19,25,29)}$, falência endotelial primária e rejeição imunológica são as principais causas de retransplante (Tabela 3).

Os transplantes de córnea realizados nos pacientes com herpes ocular representaram $6,6 \%$ dos casos, o que difere dos achados recentes da literatura americana que encontrou redução na indicação de transplante por herpes a partir da década de oitenta ${ }^{(7,23)}$. Leucoma corneano após infecção viral por herpes simples correspondeu a $5,5 \%$, herpes zoster a $0,5 \%$, úlcera neurotrófica a $0,3 \%$ e ceratite herpética ativa por herpes simples a $0,3 \%$. Nos últimos dez anos, houve aumento na frequiência de transplantes de córnea por herpes (de 3,6\% para $6,6 \%$ ), pois com a criação do ambulatório específico para atendimento de casos de herpes ocular no Setor de Córnea e Doenças Externas no Departamento de Oftalmologia da ISCMSP, nosso serviço tornou-se referência para o tratamento desta doença com aumento proporcional no número de casos atendidos.
Apesar da distrofia de Fuchs ser freqüente indicação de transplante de córnea nos EUA (com valores que variam de $13,4 \%$ a $23,8 \% \%^{(7,12,18,25,33)}$, o mesmo não foi observado em nosso serviço $(2,9 \%)$ e nos demais estudos brasileiros ${ }^{(4,13-14,16,19,25,35)}$, francês ${ }^{(23)}$, chinês ${ }^{(28)}$ e inglês ${ }^{(34)}$. Devido à menor disponibilidade de córneas no Brasil, somente pacientes com distrofia de Fuchs em fase avançada são submetidos a transplante de córnea.

\section{CONCLUSÃO}

Em nosso estudo, nos últimos 10 anos, houve aumento no número de transplantes penetrantes de córnea realizados nos casos de ceratite infecciosa (bacteriana e fúngica), trauma, CBPF e herpes e diminuição nos casos de ceratocone e retransplante, em comparação com o estudo anterior ${ }^{(19)}$.

\section{ABSTR ACT}

Purpose: To identify the indications for penetrating keratoplasty at the Department of Ophthalmology of the Santa Casa of São Paulo from January 1996 to December 2005 and to compare them with those from January 1991 to December 1995. Methods: Charts of patients who had undergone penetrating keratoplasty from January 1996 to December 2006 were retrospectively reviewed. Results: There were 587 keratoplasties done in this period. The average age of patients was $49.6 \pm 21.1$ years (range 1 to 91 years). The most common indications were infectious keratitis $(17.9 \%)$, trauma (16\%), pseudophakic bullous keratopathy $(14.7 \%)$, keratoconus (13.1\%), regrafts $(12.8 \%)$ and herpetic keratitis $(6.6 \%)$. Conclusion: The leading indications for penetrating keratoplasty were infectious keratitis, trauma, bullous keratopathy, herpetic keratitis. There was a decreasing trend in penetrating keratoplasty for keratoconus and regraft during the last 10 years.

Keywords: Corneal transplantation/statistics \& numerical data; Cornea; Keratoplasty, penetrating; Trends

\section{REFERÊNCIAS}

1. Mc Neill J. Indications and outcomes. In: Krachmer JH, Mannis MJ, Holland EJ, editors. Cornea. St. Louis: Mosby; 1997. v. 3, p.1551-61.

2. Aiken-O'Neill P, Mannis MJ. Summary of corneal transplant activity Eye Bank Association of America. Cornea. 2002;21(1):1-3.

3. Associação Brasileira de Transplante de Órgãos. Estatística de transplante [Internet].São Paulo: ABTO; s.d. [citado 2007 Dez 2]. Disponível em: www.abto.org.br

4. Cattani S, Kwitko S, Kroeff MAH, Marinho D, Rymer S, Bocaccio FL. Indicações de transplante de córnea no Hospital das Clínicas de Porto Alegre. Arq Bras Oftalmol. 2002;65:95-8.

5. Arentsen JJ, Morgan B, Green WR. Changing indications for keratoplasty. Am J Ophthalmol. 1976;81(3):313-8.

6. Smith RE, McDonald HR, Nesburn AB, Minckler DS. Penetrating keratoplasty: changing indications, 1947 to 1978. Arch Ophthalmol. 1980;98(7):1226-9. 
7. Cosar CB, Sridhar MS, Cohen EJ, Held EL, Alvim Pde T, Rapuano CJ, et al. Indications for penetrating keratoplasty and associated procedures, 1996-2000. Cornea. 2002;21(2):148-51.

8. Dobbins KR, Price FW Jr, Whitson WE. Trends in the indications for penetrating keratoplasty in the midwestern United States. Cornea. 2000;19(6): 813-6.

9. Flowers CW, Chanq KY, McLeod SD, Irvine JA, McDonnell PJ, Rao N, Smith RE. Changing indications for penetrating keratoplasty, 1989-1993. Cornea. 1995;14(6):583-8. Comment in: Cornea. 1997;16(1):120-1.

10. Patel NP, Kim T, Rapuano CJ, Cohen EJ, Laibson PR. Indications for and outcomes of repeat penetrating keratoplasty, 1989-1995. Ophthalmology. 2000;107(4):719-24

11. Randleman JB, Song CD, Palay DA. Indications for and outcomes of penetrating keratoplasty performed by resident surgeons. Am J Ophthalmol. 2003; 136(1):68-75.

12. Sugar A, Sugar J. Techniques in penetrating keratoplasty: a quarter century of development. Cornea. 2000;19(5):603-10. Erratum in: Cornea. 2001;20(1):121.

13. Ávila MP, Sugano DM, Póla CM, Lima VL, Rehder JRCL. Estatística do Banco de Olhos do ABC: 1997-2000. Arq Med ABC. 2001;24(1):56-9.

14. Cresta FB, Alves MR, Kara-Jose N, Vessani RM, Nascimento VP, Sakamoto $\mathrm{K}$, et al. Indicações de ceratoplastia em 70 pacientes estudados no Hospital das Clínicas da Faculdade de Medicina da Universidade de São Paulo no ano de 1995. Revista Médica. 1997;76(6):320-3.

15. Endris D, Cunha F, Ribeiro MP, Toscano J. Ceratoplastias penetrantes realizadas na Fundação Altino Ventura: revisão dos resultados e complicações. Arq Bras Oftalmol. 2003;66:273-7.

16. Fabris C, Corrêa ZMS, Marcon AS, Castro TL, Marcon IM, Pawlowski C. Estudo retrospectivo dos transplantes penetrantes de córnea da Santa Casa de Porto Alegre. Arq Bras Oftalmol. 2001;64:449-53.

17. Garcia EL, Netto AA, Mendes IR. Indicações para os transplantes de córnea em Florianópolis, Santa Catarina. Rev Bras Oftalmol. 2002;61(3):186-92.

18. Marchi PAH, Marchi Júnior WA, Hueb LA, Arieta CEL. Transplante de córnea no Hospital das Clínicas da Unicamp. Revista Ciências Médicas. 2002;11(2):115-22.

19.Nishiwaki-Dantas MC, Dantas PEC, Holzchuh N, Lui Netto A, Giovedi Filho R, Giovedi M, et al. Indicações de transplante penetrante de córnea. 19911995. Arq Bras Oftalmol. 1998;61(1):23-33.

20. Teixeira MF, Almeida Junior GC, Rodrigues ML, Kamimoto OS, Kashiwabuchi LK. Resultados e indicações de ceratoplastias penetrantes realizadas por médicos em treinamento, num país em desenvolvimento. Arq Bras Oftalmol. 2001;64(6):577-61.
21. Lindquist TD, McGlothan JS, Rotkis WM, Chandler JW. Indications for penetrating keratoplasty: 1980-1988. Cornea. 1991;10(3):210-6.

22. Edwards M, Clover GM, Brookes N, Pendergrast D, Chaulk J, McGhee CN Indications for corneal transplantation in New Zealand: 1991-1999. Cornea. 2002;21(2):152-5

23. Legeais JM, Parc C, d'Hermies F, Pouliquen Y, Renard G. Nineteen years of penetrating keratoplasty in the Hotel-Dieu Hospital in Paris. Cornea. 2001; 20(6):603-6.

24. Morris RJ, Bates AK. Changing indications for keratoplasty. Eye. 1989;3(Pt 4): 455-9.

25. Victor G, Barth B, Júnior AS. Avaliação das indicações em ceratoplastia penetrante. Rev Bras Oftalmol. 2002;61(3):174-8.

26. Zhang C, Xu J. Indications for penetrating keratoplasty in East China, 19942003. Graefes Arch Clin Exp Ophthalmol. 2005;243(10):1005-9.

27. Chen WL, Hu FR, Wang IJ. Changing indications for penetrating keratoplasty in Taiwan from 1987 to 1999. Cornea. 2001;20(2):141-4.

28. Sony P, Sharma N, Sen S, Vajpayee RB. Indications of penetrating keratoplasty in northern India. Cornea. 2005;24(8):989-91.

29. Oliveira LA, Corrêa BS, Almeida Jr GC, Ferrari MT, Kashiwabuchi LK. Influência da lista única de uma regional de transplantes de córnea de um Banco de Olhos vinculado a um hospital escola. Arq Bras Oftalmol. 2003; 66:631-5.

30. São Paulo (Estado). Secretaria de Estado de Saúde. Resolução SS - 103 de 01/08/97. Dispõe sobre a estrutura organizacional e operacional do Sistema Estadual de Transplantes de São Paulo. São Paulo: Diário Oficial do Estado; 1997 Fev 5.

31. Maeno A, Naor J, Lee HM, Hunter WS, Rootman DS. Three decades of corneal transplantation: indications and patient characteristics. Cornea. 2000; 19(1):7-11.

32. Yahalom C, Mechoulam H, Solomon A, Raiskup FD, Peer J, Frucht-Pery J. Forty years of changing indications in penetrating keratoplasty in Israel. Cornea. 2005;24(3):256-8

33. Kang PC, Klintworth GK, Kim T, Carlson AN, Adelman R, Stinnett S, Afshari NA. Trends in the indications for penetrating keratoplasty, 19802001. Cornea. 2005;24(7):801-3.

34. Al-Yousuf N, Mavrikakis I, Mavrikakis E, Daya SM. Penetrating keratoplasty: indications over a 10 year period. Br J Ophthalmol. 2004;88(8):998-1001.

35. Carvalho RC, Moss M, Garrido C, Cohen J, Chaves C. Indicações de transplante de córnea no Amazonas. Experiência de 11 anos no Instituto de Oftalmologia em Manaus. Rev Bras Oftalmol. 1996;55(8):619-22. 